\title{
Proceedings of the 149th Semon Club, 1 June 2015, ENT Department, Guy's and St Thomas' NHS Foundation Trust, London, UK
}

\author{
Chairperson: Miss Elfy B Chevretton, Guy's and St Thomas' NHS Foundation Trust \\ Secretary: Mr Sherif Haikel, Royal National Throat, Nose and Ear Hospital, University College London Hospitals NHS Trust \\ Invited panel for pathology: Dr Ann Sandison, Charing Cross Hospital, Imperial College Healthcare NHS Trust \\ Invited panel for radiology: Dr Steve Connor, and Dr Ata Siddiqui, Guy's and St Thomas' NHS Foundation Trust \\ Professor Leslie Michaels prize for the best presentation of the meeting was awarded to Eamon Shamil for 'Paraganglioma: the challenges of \\ peri-operative management'.
}

The chairperson and secretary have edited the proceedings of the meeting to reflect the discussion of each case by the expert panel and audience during the Semon Club meeting.

\author{
Head and neck section \\ Chairperson: Mr Richard Oakley
}

A rare cause of unilateral nasal symptoms

A Haymes, R Srinivasan, N Choudhury

East Surrey Hospital, Redhill

\section{Introduction}

Primary malignancies of the nasal cavity are rare; metastasis of infraclavicular malignancies to the nasal cavities is rarer still. Small cell lung cancer is a high-grade neuroendocrine malignancy which can rarely present as a solitary metastasis involving the head and neck.

\section{Case report}

A 58-year-old gentleman presented with a 4-month history of right-sided rhinorrhoea, hyposmia, hypogeusesthesia and right cheek hypoesthesia. Nasendoscopy demonstrated irregular ulcerated deposits within the right nasal cavity. Head and neck examination findings were otherwise unremarkable.

\section{Radiological findings}

Computed tomography (CT) scans of the head and neck demonstrated a large enhancing mass in the superior nasal cavity, which extended into the ethmoidal air cells, cribriform plate, olfactory fossae bilaterally and the anterior cranial fossa. A whole-body positron emission tomography (PET)-CT scan demonstrated right-sided cervical lymphadenopathy, extensive mediastinal and bilateral hilar lymphadenopathy, and collapse and consolidation of the right mid-lobe of the lung.

\section{Histological findings}

Biopsies demonstrated a necrotic undifferentiated tumour composed of pleomorphic epithelioid cells displaying nuclear moulding and numerous apoptoses. The appearances were consistent with a diagnosis of small cell carcinoma.

\begin{abstract}
Management
Based on histology and PET-CT results, the patient was diagnosed with stage $\mathrm{N}_{3} \mathrm{M}_{1}$ small cell lung cancer. The patient was commenced on palliative chemoradiotherapy, which achieved resolution of the nasopharyngeal mass and associated symptoms.
\end{abstract}

\section{Discussion}

Dr Connor and Dr Sandison upheld the diagnosis of small cell carcinoma with radiological evidence of a lung primary, excluding extrapulmonary small cell carcinoma as the main differential diagnosis.

\section{Conclusion}

We believe this to be the first reported case of small cell lung cancer metastasising to the nasal cavity. Immunohistochemistry and PET-CT scans proved useful in arriving at an accurate diagnosis.

Posterior commissure lesion presenting as dysphonia and progressive nocturnal wheeze

A Haymes, R Srinivasan, N Choudhury

East Surrey Hospital, Redhill

\section{Introduction}

Spindle cell carcinomas are rare malignant lesions characterised by in situ or invasive squamous cell carcinoma components combined with a spindle cell or mesenchymal component. We describe a rare case of spindle cell carcinoma of the larynx, with heterologous rhabdomyoblastic elements, which presented as dysphonia and stridor in an elderly female.

\section{Case report}

A 78-year-old Caucasian female presented to hospital with a 6-month history of dysphonia and a monophonic inspiratory wheeze. Flexible nasendoscopy demonstrated a polypoidal tumour arising from the left interarytenoid area, causing partial airway obstruction during inspiration. 


\section{Radiological findings}

Computed tomography demonstrated a posteriorly based supraglottic lesion, with normal appearances of the glottis and subglottic spaces and no evidence of locoregional spread.

\section{Histological findings}

Histology demonstrated an ulcerated polypoid tumour composed almost entirely of atypical spindle cells expressing alpha smooth muscle actin and skeletal muscle markers desmin and myogenin multifocally. No epithelial keratinisation was identified, thereby excluding a squamous cell carcinoma. According to a specialist opinion from Professor Fischer (from the Royal Marsden Hospital, London), the lesion represented spindle cell carcinoma with heterologous rhabdomyoblastic elements, rather than primary rhabdomyosarcoma.

\section{Management}

Following discussion in the head and neck multidisciplinary team meeting, the patient was clinically monitored for recurrence. At two months' post-resection, there was no macroscopic evidence of local recurrence, as confirmed on repeat biopsies. The patient continues to be closely monitored.

\section{Discussion and conclusion}

Laryngeal spindle cell carcinoma may be misinterpreted as a reactive process, particularly when the epithelial component is not readily recognisable. Rare histological features, such as rhabdomyoblastic differentiation as seen in this case, may result in the erroneous diagnosis of a primary sarcoma. Awareness of the lesion and correct diagnosis reduces the risk of inappropriately aggressive treatment that may include chemoradiation.

Recurrent cervical lymphadenopathy of unknown origin

A Patel, N Gibbins

University Hospital Lewisham

\section{Introduction}

Cervical lymphadenopathy has extensive differential diagnoses which need to be investigated, but occasionally a cause cannot be identified.

\section{Case report}

We report the case of a 34-year-old lady, who presented with a 2-year history of a slowly enlarging right neck mass. She had been experiencing night sweats and hot flushes, but no weight loss. Initial ultrasound and cytology were performed, followed by an excision biopsy. This revealed a $25 \times 20 \mathrm{~mm}$ lipoma and $35 \times 30 \mathrm{~mm}$ reactive lymph node on histology. The patient re-presented to the clinic four months later with recurrence of the mass, measuring $4 \mathrm{~cm}$, in exactly the same location. Subsequent ultrasound and cytology again showed reactive lymph node tissue only. This tissue was re-excised and sent for a secondary review. The results for autoimmune disease, toxoplasmosis, tuberculosis and human immunodeficiency virus testing were all negative.

\section{Radiological findings}

Repeat ultrasound identified a $16 \times 40 \mathrm{~mm}$ right-sided node at level II. Multiple smaller right-sided nodes, measuring up to $7 \mathrm{~mm}$, were also identified in level IV.

\section{Histological findings}

Both lymph node excision biopsies showed preserved subcapsular sinus and a follicular architecture. The appearances were consistent with florid follicular hyperplasia.

\section{Management and conclusion}

The patient was referred to the rheumatology team, who, despite arranging a multitude of investigations, have not as yet confirmed a diagnosis. The patient continues to be troubled by recurrent cervical lymphadenopathy.

\section{Discussion}

At the meeting, Mr Oakley suggested an immunological or vasculitic aetiology, particularly given the recent development of new-onset haematuria, dyspnoea and back pain. Dr Sandison reminded the audience that when cytology is negative, a lymph node should be excised and sent fresh, without formalin, to the pathologist. Dr Sandison enquired as to whether the patient had undergone dental repair or prosthetic surgery.

\section{Oral ulceration: colon, a diagnostic dilemma}

T Karunakaran, S Ali, M McGurk

Guy's and St Thomas' NHS Trust, London

\section{Introduction}

Adenocarcinoma of the oral cavity is rare. We present an unusual case of an oral tumour (which was resected) considered to be adenocarcinoma of metastatic origin.

\section{Case report}

A 71-year-old gentleman presented with a 9-month history of a persistent $1 \mathrm{~cm}$ right retromolar lesion, which caused intermittent soreness and restriction in mouth opening. His background included: prostate cancer in remission, hypertension, hypothyroidism, Barrett's oesophagus, arthritis and psoriasis, 40 pack year smoking history and alcohol intake of 20 units per week. His carcinoembryonic antigen levels were raised and prostate-specific antigen levels were normal.

\section{Radiological findings}

A positron emission tomography (PET) scan showed increased uptake in the right retromolar area. Further focal uptake was present in the ascending colon. Radiologically, this was staged as a $\mathrm{T}_{4} \mathrm{~N}_{0}$ buccal lesion.

\section{Histological findings}

Incisional biopsy findings revealed adenocarcinoma, with no appearances suggestive of oral and/or prostatic origin. Immunohistochemistry findings did not support a lower gastrointestinal origin.

\section{Management}

The patient underwent right-sided retromolar trigone resection with free-flap reconstruction, and right-sided level I-IV neck dissection. The tumour was pathologically classified as adenocarcinoma $\mathrm{T}_{1} \mathrm{~N}_{0}$ or $\mathrm{N}_{0} \mathrm{M}_{1}$. The patient will undergo gastroscopy and colonoscopy in due course.

\section{Discussion and conclusion}

Dr Steve Connor agreed that the malignant radiological characteristics were due to effacement of the pterygomandibular raphe and erosion of the mandibular ramus. He recommended an ultrasound scan of the kidneys, because occasionally renal cell carcinomas cannot be seen on PET-computed tomography scans. Dr Ann Sandison added that positivity for 
thyroid transcription factor-1 is suggestive of a lung primary, and suggested that further chest imaging might shed more light. She also pointed out that cytokeratin 7 and 20 positivity indicated a possible upper gastrointestinal or ovarian origin.

Paraganglioma: the challenges of peri-operative management

\section{E Shamil, L Brennan, P Jani}

Addenbrooke's Hospital, Cambridge University Hospitals NHS Foundation Trust

\section{Introduction}

Paragangliomas are tumours that contain chief cells derived from neural crest cells. Four per cent of head and neck paragangliomas secrete catecholamines, the majority secreting noradrenaline.

\section{Case report}

An asymptomatic 58-year-old gentleman was incidentally found to have a right-sided neck mass deep to the medial border of the right sternocleidomastoid muscle following shoulder dislocation. His blood pressure was unremarkable.

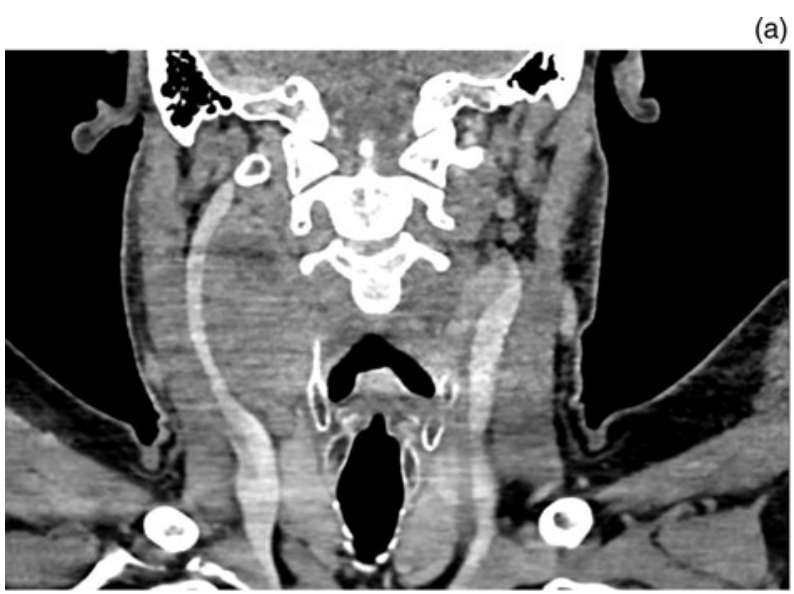

(b)

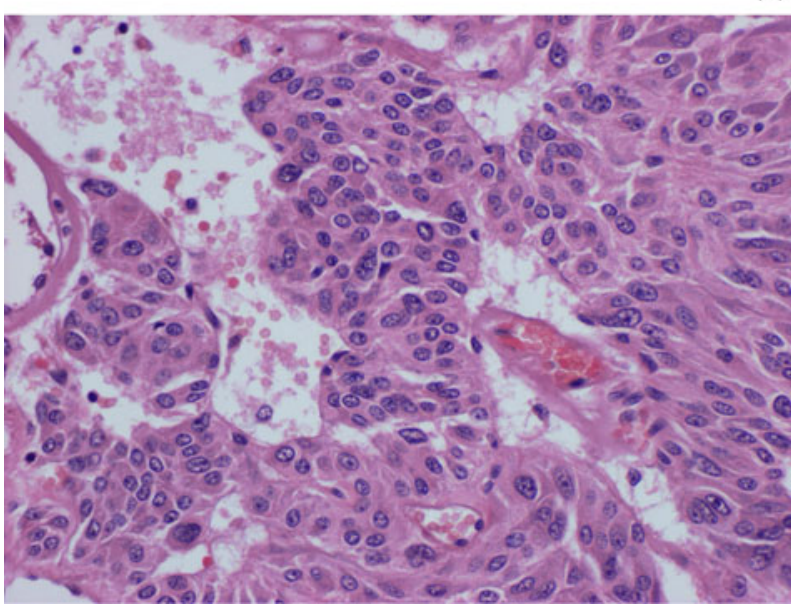

FIG. 1

(a) Coronal computed tomography scan of the neck, showing a heterogeneous lesion at the right carotid bifurcation measuring $41 \times$ $37 \mathrm{~mm}$. (b) Histology (H\&E) revealed that the tumour, arising within a nerve, was composed of oval-shaped tumour cells arranged in a nested (Zellballen) pattern.
He had raised plasma metadrenaline and normetadrenaline levels.

\section{Radiological findings}

A computed tomography (CT) scan of the neck showed a heterogeneous lesion at the right carotid bifurcation, measuring $41 \times 37 \mathrm{~mm}$ (Figure 1a). A CT angiogram showed splaying of the internal and external carotid arteries (lyre sign). Dr Connor noted the atypical location dorsal to the carotid bifurcation. Contrast revealed very little vascularity. A metaiodobenzylguanidine single-photon emission CT scan showed focal high tracer uptake in the right side of the neck.

\section{Histological findings}

The tumour was arising within a nerve composed of ovalshaped cells arranged in both nested (Zellballen) and trabecular patterns (Figure 1b). Immunohistochemistry findings were positive for neuroendocrine markers chromogranin A, synaptophysin and cluster of differentiation 56. Dr Sandison reminded the audience that cellular atypia, necrosis, mitosis and vascular invasion are only indicators of neoplasia, and not malignancy. The only indicator of malignancy was a metastatic deposit.

\section{Management}

Pre-operatively, an endocrinologist initiated alpha- and betablockers. Intra-operatively, a hypertensive crisis occurred each time the mass was manipulated. At this point, the surgeons would pause, while the anaesthetist titrated sodium nitroprusside. It would take 1-2 minutes for the blood pressure to fall to acceptable parameters. There were no post-operative complications.

\section{Discussion}

Given its location and secreting properties, Dr Connor advised that this paraganglioma may have originated from the sympathetic cervical ganglion, or it could be a lowsitting glomus vagale. He also recommended that a wholebody magnetic resonance imaging scan be performed. $\mathrm{Mr}$ Oakley enquired as to the feasibility of pre-operative embolisation to reduce blood loss and operative time. Dr Connor advised this may be challenging because of poor tumour vascularity.

\section{Rhinology section \\ Chairperson: Mr Rishi Talwar}

Transnasal endoscopic microvascular free-flap reconstruction of the skull base

\section{S Zaidi, S Parmar, S Ahmed \\ Queen Elizabeth Hospital, Birmingham}

\section{Introduction}

We report a novel procedure in which a radial forearm free flap was used to separate the nasal cavity from the brain after endoscopic excision and orbital exenteration for a squamous cell carcinoma (SCC) ex inverted papilloma. To the best of our knowledge, this is the first time this procedure has been used in the skull base in the UK.

\section{Case report}

A 61-year-old man with a history of a lateral rhinotomy (performed in 1992 to treat an inverted papilloma with a carcinoma in situ) presented in 2013 with symptoms of unilateral nasal obstruction. He underwent endonasal excision of the 
skull base with a pericranial flap inserted into the nasal cavity through a frontal slot sinusotomy for the reconstruction. He re-presented 12 months later with biopsy-proven SCC.

\section{Radiological findings}

Imaging showed an aggressive lesion involving the right medial orbital contents as well as the skull base and nasal septum.

\section{Histological findings}

Histology confirmed SCC.

\section{Management}

After endonasal skull base resection combined with orbital exenteration, reconstruction consisted of a radial forearm microvascular free flap that was pedicled to the facial artery and external jugular vein.

\section{Discussion}

Dr Sandison commented that it was an atypical papillomatous lesion, with three different histological appearances within the same specimen. There were areas of: everted dysplastic mucosa with cylindrical features, inverted dysplastic mucosa, and invasive SCC. Dr Sandison commented that it would have been interesting to review the initial histology from 1992 to see if these distinct areas were recognisable at that time; unfortunately, these slides were not available. Dr Sandison felt the lesion was best regarded as a lowgrade SCC and considered aggressive treatment to be the best course of management.

\section{Conclusion}

The microvascular free flap should be considered for skull base repair when there are no locally available flaps, especially in patients that need post-operative radiotherapy.

\section{Bilateral nasal obstruction: a very rare cause}

\section{E Akerele, A Walden, P Kothari}

Luton and Dunstable NHS Foundation Trust

\section{Introduction}

Nasal obstruction is a common symptom, often with benign aetiology. However, a thorough assessment is important to identify some of the rarer causes. We present a case of an unusual nasal manifestation of a very rare condition, with interesting radiological and histological findings.

\section{Case report}

A 55-year-old male presented with a longstanding history of progressive bilateral nasal obstruction. Endoscopic examination revealed a large septal mass almost completely blocking both nasal cavities. Given these findings, urgent radiological investigations and biopsies were performed.

\section{Radiological findings}

Computed tomography scanning of the sinuses revealed an anterior nasal mass, with erosion and destruction of the bony palate inferiorly. Subsequent magnetic resonance imaging showed an enhancing mass centred on the nasal septum and extending to the bony maxillae.

\section{Histological findings}

The lesion appeared to have a nodular architecture, and was composed of macrophages with foamy cytoplasm. Lymphocytes and plasma cells were seen singly scattered or focally in the aggregate. Large histiocyte cells showed features of emperipolesis, as highlighted by S100 protein on immunohistochemistry and staining for cluster differentiation 68 and epithelial membrane antigen. Appearances were compatible with Rosai-Dorfman disease.

\section{Management}

Following histological diagnosis, the patient was referred to and is being managed by haematologists.

\section{Semon Club discussions}

In the radiology review, Dr Connor commented on the impressive bony destruction caused by the lesion. Dr Sanderson mentioned that this disease occurs classically in the orbit.

Progressive paranasal swelling following high-pressure paint injury

J Mushtaq, A Walker, B Hunter

Croydon University Hospital

\section{Introduction}

High-pressure paint injuries are an uncommon workplacerelated injury, and most often occur to the index finger of the non-dominant hand. We present the first case report of a high-pressure paint injury occurring to the face and describe its long-term complication.

\section{Case report}

A 33-year-old gentleman presented with a progressively enlarging right paranasal swelling, which was starting to cause discomfort and impact on his vision. He had suffered a facial high-pressure paint injury eight years previously, which left extensive scarring over the right ala and dorsum of the nose.

\section{Radiological findings}

Computed tomography findings suggested a partially calcified venous malformation on the right cheek arising from the angular and facial veins. Ultrasonography revealed a subcutaneous lobulated mass with evidence of internal vascularity. However, a biopsy was advised because of difficulties in assessment and to rule out malignancy.

\section{Histological findings}

Specimens were taken intra-operatively and sent for urgent histological analysis; this showed a florid foreign body reaction to black particulate debris with colourless birefringent matter.

\section{Management}

A combined operation, which included revision of scarring and debulking of the mass with subsequent histology, was agreed upon. Access to the mass was gained through existing scars on the nose to optimise the cosmetic result.

\section{Discussion}

Dr Sandison warned that on a small biopsy, appearances could be similar to fungal invasion. Mr Talawar recalled seeing similar reactions from injectable dermal fillers.

\section{Conclusion}

High-pressure paint injuries require urgent surgical management and may lead to debilitating long-term complications. The prognosis of these injuries depends on the location of the injury, and the type, volume and pressure of the paint. 


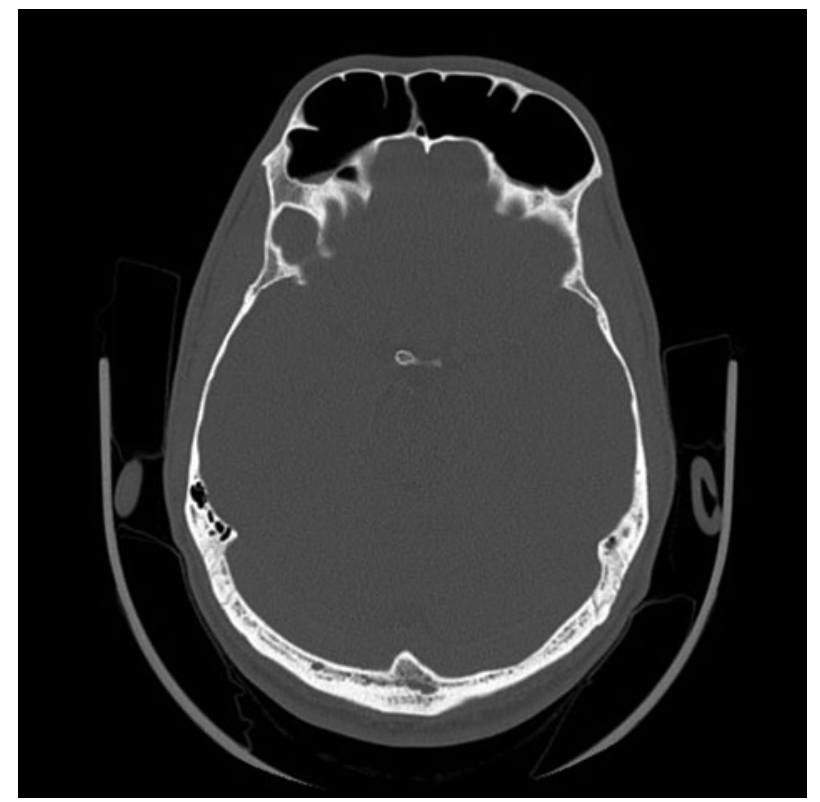

FIG. 2

Axial computed tomography scan, showing expanded frontal sinuses.

Progressively enlarging tender mass with associated skin changes

N Quraishi, S Cartwright, C Hopkins

Guy's Hospital, London

\section{Introduction}

Pneumosinus dilatans is a rare condition characterised by pathological expansion of the paranasal sinuses. It is often asymptomatic, but may cause facial bossing due to outward displacement of sinus walls.

\section{Case report}

A 25-year-old male presented to the maxillofacial surgical team with a progressively enlarging tender mass over his forehead, with associated skin changes. He gave no history consistent with rhinosinusitis. On examination, he had a large bifrontal bony swelling. Past medical history was unremarkable.

\section{Radiological findings}

Computed tomography (Figure 2) demonstrated bilateral massively expanded frontal sinuses, consistent with pneumosinus dilatans. There were associated mucosal changes suggesting that chronic sinusitis contributed to the frontal sinus enlargement.

\section{Management}

The patient underwent a Draf II frontal sinusotomy. A conservative policy is being followed to allow for spontaneous bony remodelling of the deformity.

\section{Discussion}

Balloon sinuplasty was considered; however, a formal sinusotomy was instead used to ensure maximal opening of the frontal recess.

Dr Steve Connor, consultant radiologist, suggested he would only use the term 'pneumosinus dilatans' in relation to a pneumocele. He noted extensive pneumatisation throughout the skull, which indicated a developmental aetiology rather than sinus outflow obstruction. Mr Ahmed, from Birmingham, described a similar case of developmental pneumatisation he had seen, where sinus formation had extended down the cervical spine to the level of $\mathrm{C} 3$. A developmental aetiology was thought unlikely in this case as the forehead swelling had undergone rapid enlargement over the preceding year.

The aetiologies of pneumosinus dilatans and silent sinus syndrome were discussed. The cause of expansion or contraction of sinuses is unknown, and both show a possibility of spontaneous resolution. Ultimately, these aetiologies were considered incomparable.

The velocity of psychosis

A Nassimizadeh, S Ahmed

University Hospital Birmingham

\section{Introduction}

Steroid-induced psychosis is a well-known condition. Glucocorticoids have generalised effects on cerebral blood flow, oxygen consumption and brain excitability, with emotional, behavioural and cognitive manifestations. Etomidate, first described in 1965, is a potent inhibitor of steroid synthesis, and therefore has a function in Cushing's disease.

\section{Case report}

A 51-year-old Caucasian male with a background of ill health, malnutrition and wasting presented in August 2014. The patient was suffering from Cushing's disease; a lowdose dexamethasone suppression test was positive and urinary cortisol excretion was grossly elevated. The patient was admitted with acute electrolyte disturbance, severe agitation and psychosis.

\section{Radiological findings}

Computed tomography highlighted a deviated pituitary stalk and a left-sided pituitary adenoma. Computed tomography of the thorax, abdomen and pelvis showed no ectopic source of adrenocorticotrophic hormone (ACTH) production.

\section{Histological findings}

Microscopically, there was pituitary adenoma and loosely arranged collagen full of haemosiderin indicating previous haemorrhage. The tumour stained positive for ACTH.

\section{Management and discussion}

The patient was intubated with use of etomidate and admitted to the intensive therapy unit. The patient required detention under the Mental Health Act. Cortisol reduction leads to peri-operative risk reduction. The patient subsequently underwent bilateral trans-sphenoidal adenoma resection, with complete resolution of symptoms. There was no need for post-operative hormone replacement.

\section{Otology and skull base section}

Chairperson: Miss Irumee Pai

Non-Hodgkin's lymphoma of the external auditory canal: an uncommon location and related risk factors

B A D Mettias ${ }^{1}$, H Zeitoun ${ }^{1}$, L N S Nasrallah ${ }^{2}$

${ }^{1}$ Glan Clwyd Hospital, Rhyl, Wales, UK ${ }^{2}$ Ministry of Health, Cairo, Egypt 


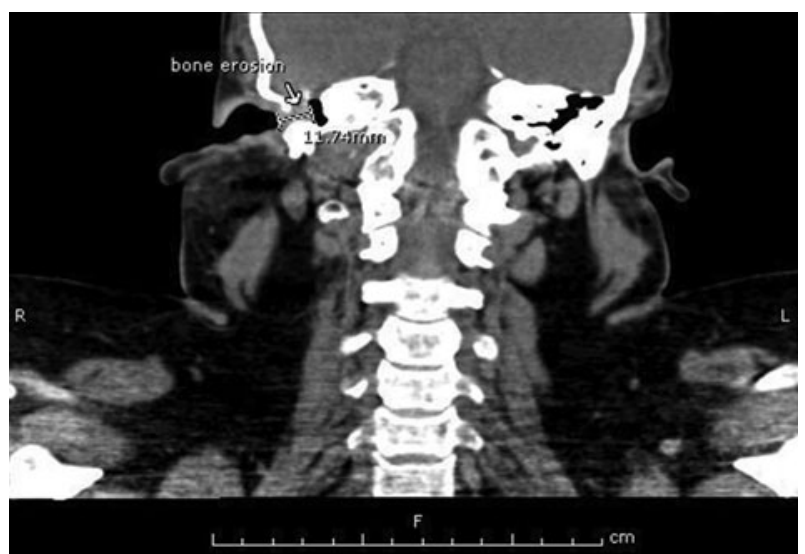

FIG. 3

Coronal computed tomography scan of the neck revealed soft tissue occluding the right external auditory canal (arrow), with destruction of the external canal roof. $\mathrm{R}=$ right; $\mathrm{L}=$ left

\section{Introduction}

The head and neck is the second most common site of primary extranodal non-Hodgkin's lymphoma, after the abdomen.

\section{Case report}

A 49-year old woman presented with a 3-week history of right-sided earache and reduced hearing. This was followed by bleeding and a rapidly progressive right facial paralysis. She had a history of hypertension, type II diabetes, refractory asthma, iatrogenic Cushing's syndrome, osteoporosis and secondary adrenal insufficiency.

The external ear canal was occluded with a reddish-purple soft tissue mass. The facial nerve paralysis was classified as House-Brackmann grade V. Full blood count and C-reactive protein levels were normal. The patient had a conductive hearing loss of less than $10 \mathrm{~dB}$.

\section{Histology}

Six biopsies showed large atypical lymphoid infiltrates. The lymphoma panel revealed a diagnosis of diffuse, high-grade, large B-cell non-Hodgkin's lymphoma.

\section{Radiology}

Imaging revealed soft tissue occluding the right external auditory canal, with destruction of the external canal roof (Figure 3). A whole-body computed tomography (CT) scan was used to classify the disease as Ann Arbor stage 1A.

\section{Management}

The patient received three courses of 'R-CHOP' (rituximab, cyclophosphamide, doxorubicin, vincristine, prednisolone) chemotherapy regimen every three weeks. The otalgia, facial palsy and hearing improved after the first course, with complete resolution after the second course.

\section{Discussion}

Dr Siddiqui suggested facial nerve injury outside the temporal bone and recommended a positron emission tomography-CT scan for staging. The acute, progressive facial palsy, with a mass in the external ear, favoured an early biopsy, especially given the normal inflammatory markers. Dr A Sandison warned of the risks of missing a lymphoma on a small crushed biopsy, and stated that a core biopsy is normally preferable.
A rare cause of unilateral pulsatile tinnitus

S Mahalingam, S Khemani

East Surrey Hospital, Redhill

\section{Introduction}

We present a case of capillary haemangioma of the tympanic membrane.

Case report

A 49-year-old gentleman presented with a 2-month history of right-sided pulsatile tinnitus. Otoscopy revealed a vascular lesion affecting the anterosuperior aspect of the tympanic membrane. A pure tone audiogram showed mild rightsided conductive hearing loss.

\section{Radiological findings}

Magnetic resonance imaging (MRI) of the head, and computed tomography (CT) angiograms and venograms were unremarkable.

\section{Histological findings}

Dr Sandison demonstrated small blood vessels and pleomorphism, suggestive of a lobular capillary haemangioma.

\section{Management}

The patient underwent exploratory tympanotomy and complete excision of the lesion. Two weeks post-operatively, his symptoms had resolved completely.

\section{Discussion}

Although the radiology findings were initially reported as normal, Dr Siddiqui highlighted thickening of the right tympanic membrane in comparison to the left, which is suggestive of pathology.

From the general discussion in this forum, it became evident that capillary haemangiomas of the tympanic membrane behave differently to those found elsewhere in the head and neck region, and they tend to be single as opposed to multiple. The six cases reported in the literature all occurred in adults with an average age of 50 years. The efficacy of beta-blockers is unknown. Dr Siddiqui recommended that patients with pulsatile tinnitus be investigated with pre- and post-contrast imaging (CT or MRI) with arterial and venous sequences.

\section{Conclusion}

Despite being a rare entity, capillary haemangioma should be considered as a possible diagnosis in patients presenting with unilateral tinnitus and abnormal otoscopy findings. Surgical excision can result in complete resolution of symptoms. Preoperative embolisation should be considered in all cases.

Infiltrative mass of the skull base and nasopharynx: a diagnostic conundrum

M George, J Goswamy, R Bhalla

Manchester Royal Infirmary

\section{Introduction}

Inflammatory skull base masses are rare and often behaviourally unpredictable. We present a case of idiopathic hypertrophic pachymeningitis forming a central skull base mass. This is the first description of mass forming or tumefactive idiopathic hypertrophic pachymeningitis extending into the nasopharynx. 


\section{Case report}

A 32-year-old woman presented with worsening headaches, serosanguinous discharge and bilateral ophthalmoplegia. Imaging confirmed a destructive skull base lesion. Cerebrospinal fluid, blood tests, and transnasal biopsies for histology and microbiology were negative. Further imaging after worsening neurology demonstrated progressive disease.

\section{Radiological findings}

Magnetic resonance imaging confirmed an extensive, destructive central skull base soft tissue mass involving the posterior clivus, floor of sphenoid sinus and nasopharynx. It extended into both cavernous sinuses, compressing both internal carotid arteries.

\section{Histological findings}

Two endoscopic biopsies demonstrated reactive lymphoid hyperplasia, which may have indicated an acute response to infection. There were no features suggestive of lymphoma, granuloma or other malignancy. Bacterial and fungal cultures were negative and antibiotics remained ineffective.

\section{Management}

Early corticosteroid treatment demonstrated radical improvement, although an initial reducing regime resulted in significant rebound deterioration. The patient was stable when discharged on a slowly reducing dose of oral prednisolone and azathioprine.

\section{Discussion and lessons learnt}

Dr Siddiqui highlighted the aggressiveness of the lesion and suggested that a diagnosis of 'inflammatory pseudotumour' might be more appropriate, but agreed that the disease may be classified as tumefactive idiopathic hypertrophic pachymeningitis.

\section{Conclusion}

For destructive skull base lesions, timely and extensive investigation is critical to reduce the risk of morbidity associated with extensive procedures. Prompt biopsy is essential to exclude multiple differentials, and early corticosteroid administration is necessary to limit local infiltration.

\section{An unusual cause of a persisting unilateral middle-ear effusion}

\section{W Ahmed, S Maskell, I Pai}

Guy's and St Thomas' NHS Foundation Trust, London

\section{Introduction}

The diagnosis of otitis media with effusion (OME) is based on history, otoscopy and audiometry. We present a patient with apparent unilateral OME, who was found to have a dural arteriovenous fistula, an associated encephalocele and middle-ear cerebrospinal fluid. This case illustrated the high index of suspicion required for an uncommon pathology when initial findings may suggest a straightforward diagnosis.

\section{Case report}

A 53-year-old man presented with a 2-year history of rightsided hearing loss and aural fullness. He had undergone ipsilateral ventilation tube insertion 10 years earlier, which resulted in immediate improvement. However, the symptoms returned on tube extrusion. Otoscopy revealed an atelectatic right tympanic membrane with middle-ear effusion, and post-auricular tenderness with pulsatility on palpation. Tympanometry confirmed right-sided middle-ear effusion.

\section{Radiological findings}

Computed tomography showed numerous occipital and mastoid bone punctate and tubular lucencies, and ossicular erosion. There was soft tissue opacification of the right middle ear, with attenuation of the right tegmen tympani and bone adjacent to the proximal tympanic facial nerve canal. Gadolinium-enhanced magnetic resonance angiography demonstrated vascular structures traversing the right occipitomastoid bones. Magnetic resonance imaging (MRI) revealed an encephalocele herniating through the tegmen defect. Conventional angiography confirmed a transversesigmoid (Cognard type I) dural arteriovenous fistula. Dr Siddiqui demonstrated the appearance of an empty sella, and suggested that maybe hydrocephalus was causing this appearance.

\section{Management}

The neurovascular multidisciplinary team meeting review suggested conservative management of the dural arteriovenous fistula. The patient awaits surgical repair of the encephalocele.

\section{Discussion}

In cases of apparent unilateral OME in an adult, every effort should be made to ascertain the aetiology, including rare but potentially life-threatening pathology. Mr Talwar enquired as to the necessity of performing an MRI scan and nasopharyngeal biopsy in all adults with a unilateral middle-ear effusion. Miss Pai stated that she does not conduct these investigations routinely if the nasopharynx looks normal on examination. Dr Siddiqui reminded the audience that an MRI scan taken with $5 \mathrm{~mm}$ slices will miss any lesions smaller than $5 \mathrm{~mm}$.

\section{An unusual third window lesion}

S Kottapalli, T Meehan, T Taylor

Royal Derby Hospital

\section{Introduction}

Cavitating cochlear otosclerosis is a rare condition, with only five case reports in the literature. We present a case of cochlear otosclerosis with a cavitating variant on the right. The unusual features in this case were that the patient had mild sensorineural hearing loss, and presented with predominantly vestibular and third window symptoms.

\section{Case report}

A 35-year-old male presented with a 1-year history of episodic disequilibrium and headaches. He complained of unsteadiness when he was upright, especially when he turned. He had difficulty hearing in background noise and experienced occasional distortion of his own voice. At follow up, he complained of worsening unsteadiness, now with sound and pressure triggers. He had episodes of pulsatile tinnitus in both ears, and autophony. He could occasionally hear his own heartbeat, breathing and eyeball movement.

\section{Audiovestibular tests}

Pure tone audiometry revealed mild sensorineural hearing loss on the right side. Otoacoustic reflexes and speech audiometry findings were normal.

Caloric testing and videonystagmography findings were normal. Vestibular-evoked myogenic potentials were inconclusive. 
Radiological findings

High-resolution computed tomography (CT) showed a curvilinear lytic lesion in continuity with the right internal auditory canal, partially encircling the basal turn of the cochlea on the right. A thin sclerotic rim was present between the abnormality and the cochlea. There was a less pronounced but similar abnormality surrounding the left cochlea (Figure 4).

\section{Management}

The patient declined vestibular rehabilitation and was managed conservatively.

\section{Discussion and lessons learnt}

Dr Siddiqui prefers the term otospongiosis to otosclerosis. He said that most cases he has seen have had both fenestral and cochlear otospongiosis. He was uncertain about the significance of the lesion on the left. High-resolution CT is indicated in all patients presenting with third window symptoms.

Dr Sandison recalled Professor Michael's theory that this condition may be a neoplastic process that begins under the periosteum, but the difficulty in acquiring biopsies made this difficult to confirm. She also preferred the term otospongiosis.

\section{Paediatric section}

Chairperson: Mr Ian Hore

Progressive unilateral facial nerve palsy in a child with contralateral facial swelling

P Bijoor, J Nichani

Manchester Royal Infirmary

\section{Introduction}

This report highlights an unusual presentation and diagnostic dilemma in a child with progressive facial nerve weakness and a contralateral facial mass.

\section{Case report}

An 11-year-old girl presented with slowly progressive right facial palsy, over 2 years. Initial magnetic resonance imaging (MRI) of the head and computed tomography scans of the temporal bones were normal. Subsequently, the patient noticed a painless lump overlying her left zygoma. An ultrasound scan and ENT review were requested. Fine needle aspiration cytology was performed, and craniofacial MRI with contrast was requested. A multidisciplinary team review, involving genetics, ENT and maxillofacial surgeons, was arranged. Given the diagnostic dilemma, it was decided to proceed to excision biopsy and carry out genetic testing for neurofibromatosis.

\section{Radiological findings}

An ultrasound scan showed an unusual soft tissue mass over the left zygoma, possibly neuroma or neurofibroma. Craniofacial MRI showed a well-defined soft tissue lesion over the left zygoma with enhancement of the underlying zygoma.

\section{Histological findings}

Fine needle aspiration cytology revealed spindle cells with some atypia. Excision biopsy of the lesion indicated nodular fasciitis.

\section{Management}

The lesion was completely excised at the initial biopsy via an intra-oral approach. Genetic tests were negative.

\section{Discussion and lessons learnt}

This case was challenging given the potential genetic implications (neurofibromatosis type 2, especially mosaicism), the suspicion of malignancy and the potential of iatrogenic facial nerve injury. Dr Sandison emphasised the importance of an excision biopsy (as compared to a core biopsy) to assess full architecture and safely exclude malignancy.

\section{Conclusion}

Nodular fasciitis is a rare, benign, rapidly growing lesion that is often misdiagnosed as malignancy. It cannot be distinguished on radiological findings and requires excision biopsy for accurate diagnosis.

\section{A rapidly enlarging right cervicofacial mass in a two-month-old child}

\section{K Evans, V Possamai}

Evelina London Children's Hospital, Guy's and St Thomas' NHS Trust

Neck masses are an uncommon presentation in children, and are generally inflammatory, neoplastic or congenital. When a vascular tumour is suspected, infantile haemangioma is the commonest form and occurs in infants.

A systemically well two-month-old girl presented to clinic with a right-sided neck mass that had rapidly grown in size over three weeks. Examination revealed a large $(5 \times 4 \mathrm{~cm})$, soft, non-fluctuant, non-tender mass overlying the parotid region, extending post-aurally and inferiorly to the submandibular region.

An initial ultrasound scan revealed a solid mass that was separate from the thyroid and parotid. It was reported as likely to be a haemangioma, but further imaging was suggested. Magnetic resonance imaging showed extension to the pre-styloid parapharyngeal space (Figure 5). The appearances were atypical for a haemangioma and warranted biopsy correlation.

Biopsy findings revealed negative microbiology cultures, with no acid-fast bacilli. Microscopic appearances were consistent with an infantile haemangioma. Therefore, propranolol therapy was initiated. Further samples were sent for glucose transporter 1 staining, which was negative, throwing the diagnosis into doubt. Samples were sent on for more specialised investigation; this confirmed kaposiform haemangioendothelioma.

Kaposiform haemangioendothelioma is a rare, locally invasive vascular tumour of intermediate histological grade, for which propranolol treatment is on occasion effective as a monotherapy. Haematology input was required as the condition is associated with a consumptive coagulopathy named Kasabach-Merritt syndrome. Further specialist advice was sought at the Great Ormond Street Children's Hospital head and neck multidisciplinary team meeting. The patient has responded well clinically, with a reduction in the size of the lesion. Therefore, propranolol remains the current treatment strategy.

\section{Laryngeal amyloidosis in a child}

\section{E Shamil, B Fish, N Jonas}

Addenbrooke's Hospital, Cambridge University Hospitals NHS Foundation Trust 


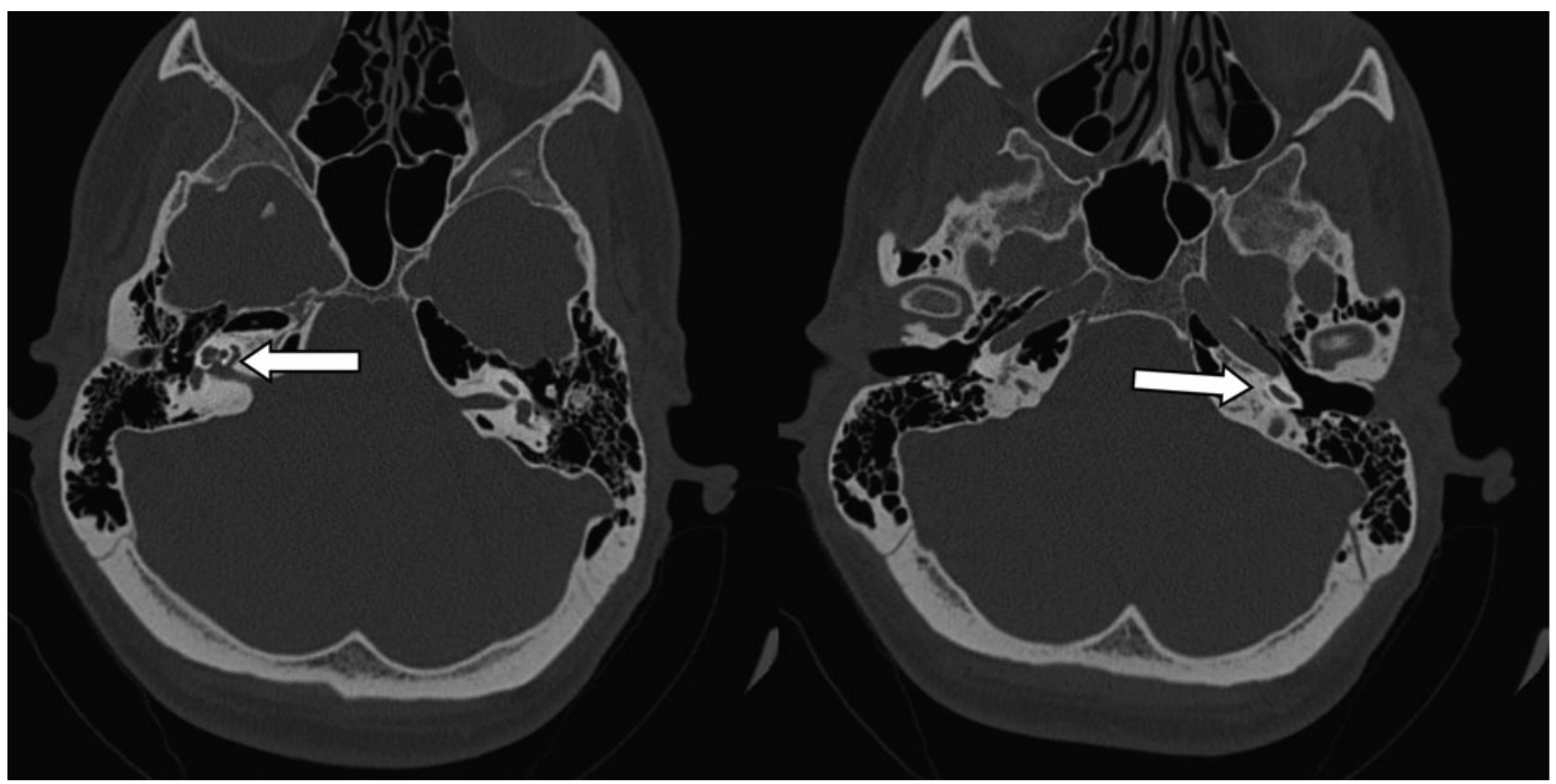

FIG. 4

Axial, high-resolution computed tomography (CT) images $(0.625 \mathrm{~mm}$ reconstructions from spiral $\mathrm{CT}$ acquisition) showing a curvilinear lytic lesion in continuity with the right internal auditory canal, partially encircling the basal turn of the cochlea on the right (arrow). A thin sclerotic rim was present between the abnormality and the cochlea. There was a less pronounced but similar abnormality surrounding the left cochlea (arrow).

\section{Introduction}

Laryngeal amyloidosis is a rare, progressive condition accounting for less than 1 per cent of benign laryngeal tumours. It is usually localised, but may be systemic.

\section{Case report}

An eight-year-old girl presented with a six-month history of snoring, noisy breathing and dysphonia. Examination revealed grade 3 tonsils (wherein the tonsils occupied

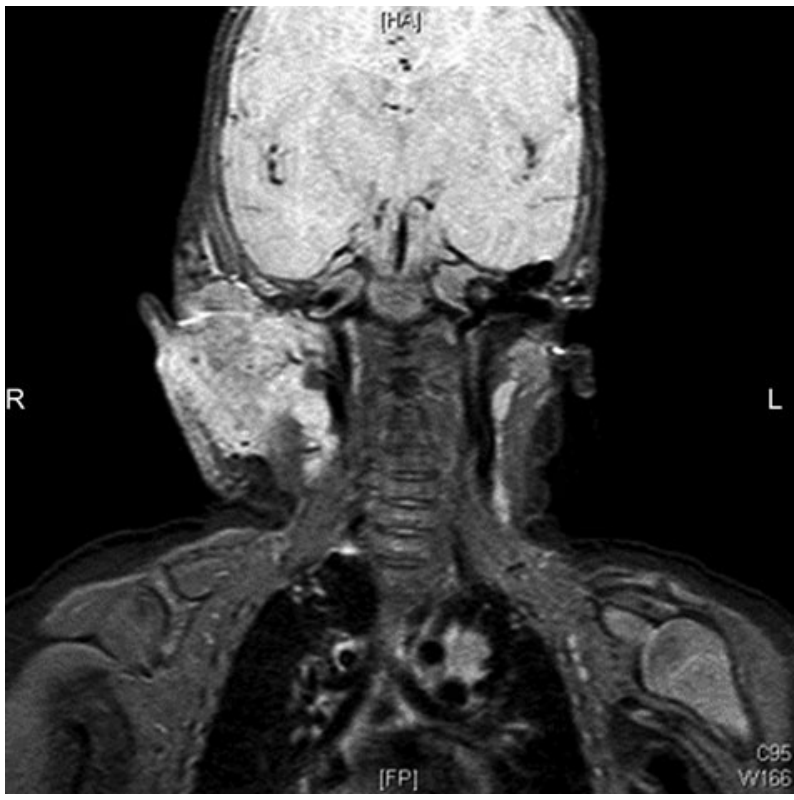

FIG. 5

Coronal magnetic resonance imaging scan, showing extension to the pre-styloid parapharyngeal space. $\mathrm{R}=$ right; $\mathrm{L}=\mathrm{left}$ between 50 and 75 per cent of the pharyngeal space). A sleep study confirmed obstructive sleep apnoea. Diagnostic microlaryngoscopy revealed a large, right-sided supraglottic mass that compromised the airway.

\section{Radiological findings}

Post-operative computed tomography of the neck showed a right supraglottic submucosal mass involving the aryepiglottic folds and false vocal cord. This narrowed the supraglottic airway. No other masses or abnormal lymphadenopathy were observed in the upper aerodigestive tract.

\section{Histological findings}

Biopsies demonstrated tissue grossly expanded by acellular hyaline eosinophilic fibres. These stained positively with Congo red and produced apple-green birefringence under polarised light.

\section{Management}

The mass was biopsied and debulked. Rheumatology input following normal investigations, which included full blood count, biochemistry, anti-nuclear antibody, anti-neutrophilic cytoplasmic antibodies, rheumatoid factor, immunoglobulins, urine electrophoresis and echocardiogram, confirmed localised (non-systemic) laryngeal amyloidosis.

Recurrence of snoring and voice symptoms three months later resulted in further debulking (using a laryngeal microdebrider) and adenotonsillectomy. This improved the patient's voice and breathing.

\section{Discussion}

Future aims include retaining a normal functioning larynx and minimising the number of debridements. Debulking using a laser is an alternative to microdebridement, as suggested by $\mathrm{Mr}$ Talwar. Miss Chevretton and Dr Sandison recommended referral to the National Amyloidosis Centre at the Royal Free Hospital in London. 


\section{Conclusion}

Laryngeal amyloidosis requires multidisciplinary management to rule out systemic involvement. Diagnosis requires positive Congo red staining producing apple-green birefringence under polarised light.

\section{Stridor and central neck mass at birth}

R Talwar, R Simo, S Blaney

Evelina London Children's Hospital, Guy's and St Thomas' NHS Trust

\section{Introduction}

Teratoid cysts are a form of dermoid, containing elements of ectoderm, mesoderm and endoderm. They occur along lines of embryological fusion. Treatment entails complete surgical excision. We describe a rare case of cervical teratoma causing significant airway compromise presenting at birth.

\section{Case report}

A male neonate was born in good condition, at term, but became cyanotic 2 minutes after birth. On airway positioning, he was observed to have a neck mass and was transferred to the neonatal intensive care unit. He had a $5 \mathrm{~cm}$, heterogeneous, non-transilluminable, central neck mass, causing positional biphasic stridor and desaturations. Fibreoptic laryngoscopy showed that the epiglottis was pushed postero-superiorly.

\section{Radiological findings}

Ultrasound appearances suggested lymphangioma, demonstrating a midline, predominantly cystic mass, with no retrosternal extension. However, magnetic resonance imaging showed a $43 \times 32 \times 35 \mathrm{~mm}$ mass, consisting of multiple cysts with intervening areas of soft tissue. The trachea was effaced, with near occlusion by anteroposterior compression. This suggested a teratoma, with significant airway compression.

\section{Management}

Surgical excision and microlaryngobronchoscopy were undertaken. The tumour was found just deep to platysma and was carefully dissected out. A small Redivac ${ }^{\text {тM }}$ drain was placed and the wound was closed in layers. Microlaryngobronchoscopy showed an improved airway, with much less narrowing of the subglottis. The patient was extubated without stridor and continued normalising thereafter.

\section{Histological findings}

Dr Sandison demonstrated a well-encapsulated lesion with cystic and solid components, with a mixture of mature tissues comprising foci of skin, fat and glial tissue. Some small follicles in keeping with thyroid tissue were seen in the periphery. Overall, the features were those of a fully excised, benign, mature cystic teratoma.

\section{Discussion}

Mr Hore advised that midline lesions causing airway obstruction could be aspirated in an attempt to deflate them. 